\title{
Pratiques et problématiques de recherche et communication environnementale : explorer de nouvelles perspectives
}

\section{Françoise Bernard}

\section{QpenEdition}

\section{Journals}

Édition électronique

URL : http://journals.openedition.org/communicationorganisation/1275

DOI : 10.4000/communicationorganisation. 1275

ISSN : $1775-3546$

Éditeur

Presses universitaires de Bordeaux

Édition imprimée

Date de publication : 1 juin 2010

Pagination : 79-89

ISBN : 978-2-86781-710-6

ISSN : 1168-5549

\section{Référence électronique}

Françoise Bernard, «Pratiques et problématiques de recherche et communication environnementale explorer de nouvelles perspectives », Communication et organisation [En ligne], 37 | 2010, mis en ligne le 02 mai 2011, consulté le 01 mai 2019. URL : http://journals.openedition.org/

communicationorganisation/1275; DOI : 10.4000/communicationorganisation.1275 


\title{
Pratiques et problématiques de recherche et communication environnementale : explorer de nouvelles perspectives
}

\author{
Fronçoise Bernord'
}

Les travaux de recherche-action que nous conduisons depuis une dizaine d'années (Bernard, 2006, 2007, 2008, 2009; Joule, Girandola, Bernard, 2007) constituent une contribution aux études consacrées à la relation entre communication et action, mais aussi à la communication de changement (sur ce thème, cf. Carayol, 2004). Ces recherches-actions sont déclinées dans trois directions principales: l'environnement, la culture et la santé. Elles sont regroupées dans un domaine d'études que nous intitulons: « la communication d'action et d'utilité sociétales ». Ces travaux intègrent l'étude de formes interorganisationnelles inédites, caractérisées par des pratiques interculturelles dans l'acception de cultures de professions et de groupes variés. Tout se passe en effet comme si la complexité des questions posées — notamment par le défi du changement climatique en ce qui concerne l'environnement, par le défi de la prévention face aux nouveaux virus (celui du sida, notamment) en ce qui concerne la santé, par le défi de la démocratie et de la création culturelles - nécessitait de penser, d'agir, de s'organiser et surtout de communiquer différemment pour apporter des réponses individuelles et collectives appropriées à ces questions. Les projets de recherche et les questions scientifiques qui sont associés à ces enjeux de société cruciaux, pris dans leur ensemble, constituent désormais un programme de recherche centré sur l'heuristique de l'action.

Ce programme et les questions qu'il soulève apportent également une contribution communicationnelle à la réflexion large et pluridisciplinaire consacrée aux approches du DD, notamment en questionnant la relation

01. Françoise Bernard est Professeur en Sciences de l'Information et de la Communication à I'Université de Provence (Aix-Marseille I). Elle est co-responsable de l'Institut de Recherche en Sciences de l'Information et de la Communication (IRSIC). Elle a présidé la Société française des Sciences de l'Information et de la Communication dont elle est actuellement Présidente d'honneur. Elle dirige le Master Information-Communication de l'Université de Provence. Elle est l'auteur de nombreux articles et chapitres d'ouvrages consacrés à la communication environnementale et à la communication de changement. Mail : francoise.bernard@ univ-provence.fr 
entre discours, action et institutionnalisation, mais aussi entre acteurs, cultures et connaissances hétérogènes. Parmi les projets qui s’inscrivent dans ce programme, nous allons donc nous centrer plus particulièrement sur les travaux consacrés à la communication environnementale.

Comment communiquer pour agir effectivement et concrètement, telle est, la plupart du temps, la demande des pouvoirs publics mais aussi plus largement des membres de la société civile (associations, ONG, entreprises) lorsqu'ils s'adressent à des chercheurs en communication.

Penser le changement en actes et la relation entre action, sens et lien, tel est le projet des chercheurs impliqués dans l'étude du paradigme de la communication engageante et instituante ${ }^{2}$. L'originalité de ce paradigme réside d'abord dans une différence de présupposé initial. Traditionnellement, dans les campagnes d'information et de sensibilisation des publics, et plus généralement dans les pratiques de communication, le présupposé selon lequel les «attitudes ", les «représentations $»^{3}$, bref les idées sont à l'origine des comportements est assez unanimement partagé. Or, chacun d'entre nous peut mesurer, au quotidien, combien le chemin qui mène des bonnes idées aux bonnes pratiques est souvent une voie longue et difficile, si ce n'est une voie sans issue. Nous sommes informés, mieux encore nous savons, et pourtant les comportements ne suivent pas, ne changent pas. C'est vrai, pour toutes les activités humaines et à différentes échelles, pour l'entreprise, la cité, le quartier, la famille, l'individu.

Les chercheurs, impliqués dans ce paradigme, prennent appui sur les études, très nombreuses, conduites aux États-Unis notamment, qui montrent que les démarches de sensibilisation portées par les campagnes de communication, si elles conduisent, dans le meilleur des cas, à des modifications dans les représentations et les opinions, n'ont pas d'effet sur les comportements effectifs. Parmi celles-ci, nous en citerons une, emblématique, consacrée aux méfaits du tabagisme et aux comportements tabagiques chez les jeunes - 8000 sujets concernés - (Peterson \& alii, 2002). Ces études montrent que les effets des campagnes d'information et de sensibilisation modifient certaines représentations et connaissances mais n'ont pas d'impact sur les

02. Le paradigme de la communication engageante a, dans un premier temps, été défini avec l'application de la théorie de l'engagement aux enjeux de la communication publique (cf. l'article fondateur : Joule, R.-V. (2000). Pour une communication organisationnelle engageante: vers un nouveau paradigme. Sciences de la Société, 50-51, 279-295) ; il a, dans un deuxième temps, été retravaillé et élargi avec et par la coopération entre chercheurs en Psychologie sociale et chercheurs en SIC (projets de recherche financés et publications) ; cette deuxième étape (cf. notamment : Bernard \& Joule 2005) marque l'émergence de la communication engageante ET instituante. II s'agit de penser théoriquement et pratiquement la relation entre communication et changement en actes associée à la question de l'institutionnalisation de nouvelles valeurs, significations et normes sociales. Cette institutionnalisation implique la question communicationnelle de la publicisation telle qu'elle est renouvelée par les usages des nouveaux médias.

03. Ces deux notions -attitudes et représentations- renvoient à des notions fréquemment citées en SIC mais qui ont été forgées dans le champ de la psychologie sociale (cf. : Vallerand R.V (1994). Les fondements de la psychologie sociale, Gaëtan Morin Ed. ; ou une version plus complète: Beauvois J.-L. La psychologie sociale, 6 tomes, Grenoble: PUG). 
comportements. En partant de ce constat, des chercheurs en SIC et en psychologie sociale qui travaillent sur le thème du changement en actes explorent dans une logique pluridisciplinaire depuis une dizaine d'années une autre voie théorique et pratique et partent du présupposé selon lequel la production des actions à un moment donné est liée aux actes qui ont été précédemment réalisés.

\section{La communication comme mise en relation entre micro - méso - macro - niveaux d'analyse et de pratiques}

Les chercheurs en Sciences de l'information et de la communication (SIC) étudient le rôle et la place de la communication dans la construction de la société, celle-ci est à la fois productrice et produit de relations complexes entre macro - méso - micro niveaux. Cette mise en relation est un objet d'études mais aussi une question méthodologique et théorique qui concerne autant les sociologues contemporains que les spécialistes de la communication.

Le paradigme de la communication engageante et instituante, conçu par des chercheurs en Psychologie sociale et en SIC (Bernard et Joule, 2005), propose une voie originale pour penser cette mise en relation entre macro -méso-micro niveaux d'analyse et de pratiques. Ce paradigme s'inscrit dans une perspective, présente différemment dans le champ de la sociologie également (cf. notamment: Touraine, 1984; Callon, Lascoumes \& Barche, 2001), qui consiste à considérer qu'une société est aussi le produit de l'action des humains, ceux-ci agissant, pour les chercheurs en communication, dans des situations de communication avec leurs valeurs, leurs stratégies et leurs cultures.

Les chercheurs en communication engageante et instituante contribuent à la conception de dispositifs, dans des situations de communication participative avec d'autres partenaires (pouvoirs publics, associations, entreprises), qui ouvrent un cours d'action à partir d'un acte préparatoire (Freedman \& Fraser, 1966; Joule \& Beauvois, 2002). L'acte préparatoire consiste à proposer un petit acte peu coûteux (en temps, en énergie, en argent...) qui favorise, comme le montre la littérature, la réalisation d'autres actes qui vont dans le même sens. Ce principe du primat de l'action est pris et repris dans des «matières signifiantes " (Veron, 1987) qui contribuent à la production de nouvelles valeurs, significations et normes sociales; c'est pour rendre compte des travaux en cours consacrés aux relations complexes entre action - interaction - signification - institutionnalisation - que la notion de communication instituante complète celle de communication engageante. Dans cette perspective, les chercheurs étudient certains passages, par exemple le passage entre significations individuelles et significations collectives, le passage entre action/cognitions individuelles ET actions/cognitions collectives. Il s'agit aussi de comprendre le passage entre communication engageante et communication instituante à l'œuvre dans ces recherches-actions. Parmi ces passages, trois d'entre eux sont particulièrement représentatifs des travaux conduits. 
1- Le passage entre action et cognition pour l'individu - qui s'opère autour des notions de rationalisation en actes et « d'identification de l'action » (Wegner et Vallacher, 1984) - fait l'objet d'applications dans des productions médiatiques et productions d'objets de communication pour les projets de recherche en communication engageante. Par exemple, la campagne d'affichage qui a accompagné le projet centré sur les économies d'énergie dans une Ville moyenne du Var (projet financé par le Conseil de Région PACA avec le soutien de l'ADEME) a été déclinée sur le plan sémiotique (image et écrit) en trois niveaux d'identification de l'action. Ces trois niveaux peuvent être résumés de la manière suivante: niveau 1 "si chacun économise un peu » en réalisant un écogeste (éteindre une lampe, fermer un robinet), niveau 2 «ma Ville devient davantage écoresponsable » et, niveau 3 « la planète respire mieux " Ces affiches conjuguaient trois niveaux d'identification de l'action: l'écogeste, la responsabilité écocitoyenne dans la cité et une conscience de l'interdépendance entre l'action humaine et la biosphère à l'échelle planétaire.

2- Le passage, dans une situation de communication engageante, entre l'engagement individuel - qui s'opère autour de l'acte préparatoire et qui peut conduire à une action socialisée favorisée par le caractère public de l'acte - est double. Il concerne d'abord l'action ponctuelle vers une action plus systématisée et pérennisée individuelle (cf. le célèbre effet de gel de Kurt Lewin, 1951); il concerne ensuite la possibilité d'une mimèsis sociale dès lors qu'il y a présence et regard de l'autre (possibilité d'un effet synchronique). On note que le passage entre un micro acte ponctuel (acte préparatoire) et un cours d'action plus stabilisé pour l'individu est favorisé par les raisons internes de l'action: «quand j'agis de cette manière, c'est conforme à ce que je suis (Joule \& Beauvois, 2002). Il s'agit aussi d'un passage diachronique qui inscrit dans la durée un acte ponctuel. Ainsi, sur les plages de Marseille, les baigneurs qui déposent un bulletin d'engagement auprès des ambassadeurs (membres d'associations environnementales), sous le regard d'autres baigneurs, peuvent éveiller curiosité et intérêt pour l'engagement à la préservation du littoral méditerranéen. Ces milliers (presque 5000 ) de baigneurs engagés contribuent, chacun, à socialiser de bonnes pratiques, en l'occurrence celles de se sentir responsables de la prise en charge des déchets sur la plage et de la préservation du littoral ${ }^{4}$.

3- Le passage entre «communication engageante » et «communication instituante» permet de théoriser ce qui est à l'œuvre, sous forme de continuités et de discontinuités, dans cette relation entre micro, méso et

04. Les projets de recherche financés, par exemple celui de « Ma plage, moi je la respecte » ont été conduits selon une méthodologie plurielle intégrant une approche expérimentale. Celle-ci permet de comparer les effets de la communication engageante (une plage A) sur le changement comportemental, la mémorisation des messages et des connaissances et le prosélytisme, aux effets d'une communication classique (sensibilisation et information sans engagement, plage B) et à une population contrôle (sans aucune intervention, plage $\mathrm{C}$ ) en veillant bien évidemment à des critères de comparaison maîtrisés (sociologie, genre, effectifs, etc.) et dans les limites de ce qui peut être fait en environnement naturel (vs laboratoire). 
macro niveaux. La dynamique instituante est favorisée par un ensemble de pratiques de médiation et de médiatisation. En ce qui concerne les pratiques de médiation, nous prendrons l'exemple des connaissances relatives au «bassin versant ", ou encore au rôle des herbiers de posidonie, diffusées lors des projets de sensibilisation des usagers (baigneurs et plaisanciers) du littoral méditerranéen (projets de recherche financés par l'ADEME et le Conseil de Région PACA). En ce qui concerne les pratiques de médiatisation, nous prendrons l'exemple de la conception et du test d'un site Internet engageant pour favoriser les pratiques d'écoconsommation, en l'occurrence l'achat et l'installation à domicile d'ampoules à économie d'énergie pour des clients d'une grande enseigne de bricolage et la mémorisation de connaissances relatives aux économies d'énergie à domicile — près de 400 sujets concernés 5 .

Pris dans leur ensemble ces "passages " illustrent des mises en relation entre pratiques et théorisations. Dans les deux cas, ils renvoient à une pensée qui favorise la mise en ligne d'un petit acte individuel (micro niveau) avec la socialisation d'actions dans un espace-temps donné: sur la plage pendant l'été (méso niveau), et avec la généralisation et la pérennisation de connaissances et de valeurs (macro niveau).

\section{Le retour de l'acteur, de l'engagement, du politique : enjeux et ambiguîtés}

Définir la communication dans la perspective d'une théorie de l'action n'est pas si fréquent dans le champ des SIC. C'est l'orientation forte de notre programme de recherche. La question du «sens » est traditionnellement présente pour un ensemble de domaines en SIC: études médiatiques, communication et politique, usages des TIC, etc. Dans ce cadre, les références sont multiples: approche sémiotique, analyse de discours et de récits, analyse sociolinguistique de corpus, etc. La question du lien est également posée en SIC dès lors que l'on considère que la communication s'inscrit dans des formes sociales qui sont elles-mêmes tissées de pratiques de médiation et de médiatisation favorisant ou non l'unité sociale et l'intercompréhension.

La question de l'action et de ses théorisations est plutôt nouvelle en SIC comme perspective de recherche clairement revendiquée. De manière générale, la théorisation de l'action sociale est plutôt implicitement laissée aux philosophes et aux sociologues. Dans le champ de la sociologie, le retour de l'acteur, annoncé en 1984 par Alain Touraine, ne cesse de se confirmer comme préoccupation forte. Plus généralement, avec les « nouvelles sociologies », des apports théoriques proposent de faire tenir ensemble logiques d'acteur et logiques d'institution, par exemple avec la Sociologie de l'expérience (Dubet, 1994) qui croise trois logiques: l'intégration (identité et groupe), la stratégie (rationalité et utilité), la subjectivité (le Moi et les valeurs) qui conduisent à l'organisation de l'action sociale.

05. Expérimentation conduite par Courbet, Bernard \& Joule dans le cadre d'un projet financé par l'ANR, cf. Bernard, 2009. 
Le projet des chercheurs en communication engageante et instituante n'est pas de s'inscrire dans les pas des travaux sociologiques, mais plutôt de développer, d'abord, un apport spécifique qui consiste à ouvrir la «boîte noire " de l'action individuelle (qui est toujours aussi traversée par du social) et pour cela de mobiliser les ressources théoriques de la psychologie sociale (Joule \& Beauvois, 2002). Ensuite, ce projet théorise, lui aussi, l'articulation entre action et institution en montrant plutôt l'importance des pratiques de médiation et de médiatisation; ce qui revient à étudier un point aveugle dans les travaux conduits par ailleurs dans les autres sciences sociales. La réflexion théorique pluridisciplinaire (Bernard \& Joule, 2005) qui consiste à associer «action ", « institution » et «communication » apporte aussi une contribution à la question des voies de renouvellement de l'imaginaire social. Ce renouvellement porte à un premier niveau sur la reformulation de la relation entre humain et biosphère, mais surtout, et à un deuxième niveau, sur la relation entre rationalités scientifiques-techniques et projet politique, dans l'acception d'un « vivre ensemble » qui intègre la flèche du temps et donc la préoccupation du futur. Cette reformulation a par ailleurs à voir avec les grands débats philosophiques contemporains portant sur la fin d'un âge philosophique:

"Ce que découvrent à divers titres, et à des degrés divers les grandes «suites» qui constituent les philosophies symptomatiques du siècle est que le caractère illimité de la raison est inséparable du caractère illimité de l'économie et de la technique, et qu'il est de plus en plus dépourvu d'une capacité parallèle de contrôle et d'autorégulation " (Tosel, 2001, p. 13).

D'une certaine manière, on peut questionner les propositions "doxiques " (vs épistémè-philosophie) du développement durable et de l'écoresponsabilité ${ }^{6}$ quant à leur prétention à "reprendre la main ", c'est-à-dire à reprendre l'idée d'une possibilité d'autorégulation mise en pratique. Ce questionnement plaide en faveur de l'approfondissement d'une approche épistémique et pluridisciplinaire du DD. Des auteurs soulignent que ce qui est devenu un slogan, le DD, renvoie aussi à un constat qui appelle une rupture (Bourg et Rayssac, 2006). Cette rupture a à voir avec une rupture dans la pensée et dans les pratiques économiques, sociétales et culturelles mais aussi dans les paradigmes et pratiques de recherche. En ce qui concerne ces dernières, l'enjeu est de développer une pluridisciplinarité effective et exigeante accompagnée, dans une perspective d'expérimentation sociétale, d'une recherche participative associant chercheurs et non chercheurs. André Tosel avait aussi lancé un appel, dans le champ philosophique, en faveur d'un agôn qui permettrait une confrontation entre philosophes afin de "philosopher à la hauteur de la mondialisation et de sa rationalité tendanciellement irrationnelle » 06. Si la notion d'éco responsabilité doit beaucoup aux travaux d'Hans Jonas consacrés au principe responsabilité (Jonas, H. (2008). Le Principe Responsabilité - Une Éthique Pour La Civilisation Technologique. Paris: Flammarion), contrairement aux propositions de ce philosophe, dans les travaux que nous conduisons, la figure de la responsabilité est élargie à chaque sujet-citoyen, quels que soient son âge, son sexe, sa catégorie socio-professionnelle. 
(Tosel, 2001, p. 23). On peut, en déplaçant cette proposition dans le champ de la communication des organisations, se demander en quoi et comment les parties prenantes ${ }^{7}$ pourraient, à leur manière, participer également à un agôn, c'est-à-dire à un espace de confrontation entre des points de vue rivaux. Dans ce cas, la notion de parties prenantes ne serait pas cet ensemble un peu mou et flou, faussement consensuel et symétrique, mais permettrait de révèler au contraire des tensions et des contradictions qui pourraient nourrir un point de vue dialogique.

Ce que nous avons observé dans la conduite des projets que nous avons vécue est que le micro acte, par exemple la prise en charge du déchet sur la plage, est associé à des « matières signifiantes » qui favorisent des basculements vers d'autres questions plus larges synchroniquement et diachroniquement. Le déchet est ainsi perçu comme menace pour la faune et flore marines, mais aussi pour la cité (Marseille et la question récurrente de la propreté urbaine), et surtout comme symbole d'une société productiviste et consumériste (moins de déchets, c'est consommer et produire différemment). Le thème d'une meilleure maîtrise des déchets est en quelque sorte peu à peu institutionnalisé et institutionnalisant. L'organisation de la «Semaine européenne de la réduction des déchets » du 21 au 29 novembre 2009 qui a donné lieu à une mobilisation des parties prenantes et à une forte communication (www.ewwr. $\mathrm{eu} / \mathrm{fr}$ ) va dans ce sens. Plus généralement, dès lors qu'il y a doute, voire critique, portant sur le modèle productiviste et consumériste, la question devient: pour le remplacer par quel autre modèle? C'est là que la notion d'agôn prend tout son sens. Au plan théorique, la question du projet politique et social rebondit, par exemple peut-on approfondir, développer la pratique démocratique tout en l'associant à une écoresponsabilité distribuée. D'autres questions émergent. Dans ce modèle, quel serait le jeu des organisations économiques (Shareholder Model vs Stakeholder Model: cf. Clarke, 1998), quelle serait la place des technosciences, comment les citoyens pourraient-ils être mieux représentés? En ce qui concerne cette question de la représentation, celle-ci est en crise par trois bords. Le premier bord est celui de l'effondrement du modèle collectiviste, les partis ont failli dans leur mission de représentation du peuple. Le deuxième bord est celui du modèle démocratique occidental, les représentants ont perdu beaucoup de leur légitimité et de leur autorité. En même temps, le jugement du citoyen est anesthésié « par le goût utilitaire du bien-être privé » (Damien, 1993, 132), ce qui constitue le troisième bord. De plus, le modèle d'un approfondissement-renouvellement de la démocratie n'est pas seul en piste dans le paysage des théories philosophico-politiques contemporaines, d'autres penseurs, néolibéraux, vont plutôt dans le sens

07. La notion de partie prenante a été définie comme : " tout groupe ou individu qui peut affecter ou qui peut être affecté par la réalisation des objectifs de l'entreprise " (Freeman, E. R. (1984) E. R. Strategic Management: A Stakeholder Approach, Pitman, Boston). Cette notion sert à fonder une perspective théorique relevant des théories des organisations et contribuant à la définition d'un modèle relationnel de l'organisation. 
d'une «désémancipation » et d'une réduction des pratiques démocratiques (cf. notamment Hayek, 1986). Le débat qui porte sur la définition d'un bon gouvernement est donc vif. Du côté de la philosophie écologique, des travaux sont publiés qui questionnent le renouvellement démocratique au regard de ces enjeux écologiques:

"Les démocrates écologiques proposent au contraire que désormais la mesure du bon gouvernement soit sa tendance à susciter un souci intense de protection de la "nature», selon ses multiples significations. » (Bourg, 2009 c, 31).

Autant dire que l'écoresponsabilité est à la fois un problème environnemental, sociétal, économique, communicationnel ET politique. Dans la réflexion politique, on trouve l'impératif de l'action conjugué à l'approfondissement démocratique. Le retour de l'acteur, de l'engagement, du politique renvoie à des enjeux cruciaux, mais est aussi porteur d'ambiguïtés et de nouvelles questions.

\section{Conclusion - Heuristique de l'action et communication participative : vers un nouvel imaginaire?}

Les chercheurs en communication engageante et instituante adoptent une posture de chercheur engagé. Ils revendiquent un statut d'acteur du changement sociétal. Ils entendent associer des «savoirs académiques » à des pratiques sociétales partant de l'idée qu'il y a bel et bien une urgence à l'action, ce qui revient à prendre position en faveur des alertes scientifiques (sur ce point, cf. Bourg, 2009, a et b). Bien sûr, l'urgence à agir se conjugue avec la proposition de re-problématisations et de théorisations assez souvent inédites.

Cette urgence à agir conduit également à construire des formes interorganisationnelles inédites dans le cadre de chacun de ces projets de recherche. Les chercheurs en communication engageante et instituante conduisent les projets dans des relations étroites avec les pouvoirs publics, le mouvement associatif et des partenaires entreprenariaux. Ils définissent ces assemblages comme relevant du courant de la « recherche-action participative et coopérative " (Bernard, 2008).

Les recherches-actions (RA) conduites dans le cadre de ce paradigme ont donc toutes à voir avec des logiques participatives. Dans ces projets, les non chercheurs (associés à la conception de la RA), mais aussi les publics enquêtés (destinataires de la RA) sont des sujets dotés d'un statut d'acteurs partenaires. Par exemple, les membres des associations sont formés à la communication engageante pour réaliser leur intervention d'ambassadeurs sur les plages et auprès des plaisanciers. Par ailleurs, l'expertise de ces associations - qui repose sur l'expérience - est prise en compte pour opérationnaliser les projets, par exemple pour définir les modes de prise de contact avec les publics. Enfin, ces ambassadeurs se présentent aux sujets enquêtés dans la posture de: "nous avons besoin de vous...", dotant ainsi ces sujets d'un statut d'acteurs-partenaires. Au fur et à mesure que les projets se déroulent, de 
nouvelles questions se posent et des réglages et réponses sont apportés dans des logiques d'échange, de délibération mais aussi de tension. L'heuristique de l'action fonctionne ainsi à plusieurs niveaux:

- pour le premier cercle des RA (laboratoire, associations, pouvoirs publics): au niveau des pratiques de recherche associées aux pratiques sociétales et de gouvernance publique,

- pour les chercheurs: au niveau des questions que pose le terrain aux chercheurs,

- pour les sujets enquêtés: au niveau d'actions engendrant d'autres actions, des nouvelles connaissances et questions,

- pour les entreprises: au niveau d'une expérience d'entreprise citoyenne (une contribution, source de valorisation, est apportée dans une logique de parties prenantes et de RSE cf. Bernard, 2009).

Par ailleurs, l'engagement se déroule dans des situations de communication, avec caractère public de l'acte et publicisation dans des espaces publics partiels (PQR, TV régionale, Internet, etc.). Des réseaux sont construits qui débordent les capacités d'action et de publicisation de chacune des organisationsinstitutions prise séparément. Ces réseaux, et les pratiques de médiation et de médiatisation qui leur sont associées, favorisent la socialisation et l'institutionnalisation de l'action environnementale et de l'écoresponsabilité.

Les chercheurs en communication engageante et instituante questionnent actuellement la relation entre ces projets de recherche-action participatifs (Bernard,2008) pris dans leur ensemble etl'émergence d'un nouvel « imaginaire socio-historique » (Castoriadis, 1979). Cet imaginaire relativiserait la place de l'économisme (autolimitation vs croissance et hyperconsommation) au profit d'une écologie intégrée dans un projet politique d'approfondissement de la démocratie où le citoyen pourrait être davantage protagoniste dans les choix concernant la cité et l'état plus soucieux des « biens publics » inaliénables.

\section{BIBLIOGRAPHIE}

BERNARD, F. \& JOULE R.V. (2005). Le pluralisme méthodologique en Sciences de l'Information et de la Communication à l'épreuve de la communication engageante. Questions de communication, 7, 185-207.

BERNARD F., (2006). «Organiser la communication d'action et d'utilité sociétales. Le paradigme de la communication engageante ", Communication \& Organisation, GREC/O, Université Michel de Montaigne, $n^{\circ}$ 29, juin, p. 65-86.

BERNARD F., (2008). "Questionner de nouveaux enjeux symboliques pour les organisations: la communication environnementale et la "communication responsable ", MEI, n ${ }^{\circ}$ 29, Paris: L'Harmattan, p. 27-38.

BERNARD F., (2008). "La recherche-action dans les travaux consacrés à la communication d'action et d'utilité sociétales: le cas de la communication engageante et de 
l'environnement ", in Bouzon A. \& Meyer V., La Communication des Organisations: Entre Recherche ET Action, Paris : L'Harmattan, p. 145-155.

BERNARD, F., (dir. scientifique), (2008). Rapport scientifique final: «Sensibilisation des baigneurs à l'environnement marin et à la propreté urbaine sur les plages de Marseille » - «Ma plage, moi je la respecte! », commanditaires: Conseil Régional PACA et ADEME, Marseille: juin 2008, 74 p.

BERNARD, F., (2009). "Apports des recherches consacrées à la communication environnementale engageante pour penser les questions des "parties prenantes " et des "frontières organisationnelles ", Actes en ligne du $4^{\circ}$ Colloque RIODD: La RSE: une nouvelle régulation du capitalisme- http://clerse.univ-lille1.fr/spip/spip.php?article391

BERNARD, F. (coord. scient.), (2009). La communication engageante au service de l'écocitoyenneté. ANR-05-|N|T|05|1|4|4|3|8|9|

BOURG, D., \& Rayssac G.-L., (2006). Le développement durable. Maintenant ou jamais. Paris: Découvertes- Gallimard.

BOURG, D., (2009 a). L'impératif écologique. Esprit. p. 1-13.

BOURG, D., \& WHITESIDE, K., (2009 b). Pour une démocratie écologique, la vie des idées.fr, p. 1-32.

CALLON M., LASCOUMES P. \& BARCHE Y., (2001). Agir dans un monde incertain. Essai sur la démocratie technique, Paris: Seuil.

CLARKE, T., (1998). "The Stakeholder Corporation: A Business Philosophy for the Information Age ", Long Range Planning, Vol. 31, n 2, p. 182-194.

CARAYOL, V., (2004). Communication organisationnelle. Une perspective allagmatique, Paris: L'Harmattan.

CASTORIADIS C. (1979). L'institution imaginaire de la société. Paris: Seuil.

DUBET, F., (1994). Sociologie de l'expérience. Paris: Seuil.

DAMIEN, R., (1993).Tocqueville, la démocratie et le conseil de philosophie, In Tosel A., (sous la dir.) La démocratie difficile. Actes du colloque franco-italien. Besançon: Presses Universitaires de Besançon, p. 119-142.

FREEDMAN, J.-L. \& Fraser, S.-C., (1966). Compliance without pressure: the foot-inthe-door technique, Journal of Personality and Social Psychology, 4, 195-202.

FREEMAN, R. E., (1998). "A Stakeholder Theory of the Modern Corporation ", in Pincus Hartman L., Perspectives in Business Ethics, Mc-Graw-Hill, p. 171-181.

JONAS, H., (1979; 1990 trad. Franç.). Le principe responsabilité. Une éthique pour la civilisation technologique. Paris : éd. du Cerf.

JOULE, R.V, \& EAUVOIS, J.-L. (2002). Petit traité de manipulation à l'usage des honnêtes gens (nouvelle version). Grenoble: Presses Universitaires de Grenoble.

JOULE, R.V., GIRANDOLA, F. \& BERNARD, F., (2007). How can people be induced to willingly change their behavior? The path from persuasive communication to committing communication. Social E Personality Psychology Compass 1:10.1111/j.17519004.2007.00018.x, http://www.blackwell-synergy.com/toc/spco/0/0.

LEWIN, K. (1951) Field theory in social science; selected theoretical papers. D. Cartwright (ed.). New York: Harper \& Row. 
PETERSON A.V., KEALEY K.A., MANN S.L., MAREK P.M. \& SARASON I.G. 2000. Hutchinson Smoking Prevention Project: long-term randomized trial in school-based tobacco use prevention--results on smoking. // Journal of the National Cancer Institute, n ${ }^{\circ} .92$, p. 1979-1991.

ROSÉ, J.-J., (2007). Responsabilité sociale dans l'entreprise - Pour un nouveau contrat social, De Boeck.

TOSEL, A., (2003). Avant-propos. In Noesis « Formes et crises de la rationalité au XX siècle ", $\mathrm{n}^{\circ} 5$, p. 9-23.

TOSEL, A. (sous la dir.), (1993). La démocratie difficile. Actes du colloque franco-italien. Besançon : Presses Universitaires de Besançon.

TOURAINE, A., (1984). Le retour de l'acteur. Paris: Fayard.

VERON, E., (1987). La semiosis sociale. Vincennes: Presses Universitaires de Vincennes.

WEGNER, D.-M., \& VALLACHER, R.-R., 1984. The emergence of action. Journal of Personality and Social Psychology, 46, p. 269-279.

Résumé : L'auteur, à partir d'une synthèse des recherches financées qu'elle a dirigées (ANR, Conseil de Région, ADEME, ISCCnrs), propose, en questionnant la relation entre pratiques de recherche et problématiques de " communication environnementale ", de montrer que les travaux sur cette thématique sont porteurs de questions vives et fortes concernant la rencontre entre enjeux de société et enjeux de recherche. Tout se passe en effet comme si la thématique environnementale, parce qu'elle permet de révéler ambiguïtés et paradoxes - pour l'entreprise, pour les pouvoirs publics, pour l'exercice de la citoyenneté, pour la recherche - ouvre un champ de questions qui fait qu'on ne peut plus penser ni faire comme avant (tout en ne sachant pas forcément pour autant comment penser et faire différemment). Ce thème conduit à formuler de nouvelles questions ou à reformuler des questions plus classiques.

Mots-clés : communication, environnement, développement durable, action, engagement, institution, imaginaire

Abstract : The author, from a synthesis of the financed researches which she managed (ANR, Council(Advise) of Region, ADEME, ISCCnrs), suggests, by questioning the relation between practices of research and problems of "environmental communication ", showing that the works on this theme are carriers of lively and strong questions concerning the meeting between stakes in society and stakes in research. Everything indeed takes place as if the environmental theme, because she allows to reveal ambiguities and paradoxes - for the company, for the public authorities, for the exercise of the citizenship, for the research, opens a field of questions which makes that we cannot think any more nor make as before (not while knowing how to necessarily for all that how think and make differently). This subject leads to formulate new questions or to repeat more classic questions.

Keywords : Communication, environment, sustainable development, action, commitment, institution, imaginary 
